\title{
THE DETERMINATION OF THE SURFACE IMPEDANCE OF AN OBSTACLE
}

\author{
by ANDRZEJ W. KȨDZIERAWSKI*
}

(Received 21st February 1991)

\begin{abstract}
The inverse scattering problem we consider is to determine the surface impedance of a three-dimensional obstacle of known shape from a knowledge of the far-field patterns of the scattered fields corresponding to many incident time-harmonic plane acoustic waves. We solve this problem by using both the methods of Kirsch-Kress and Colton-Monk.
\end{abstract}

1991 Mathematics subject classification: 35J05, 76Q05

\section{Introduction}

The inverse scattering problem we shall consider in this paper is the problem of determining the unknown surface impedance for a given three-dimensional obstacle from a knowledge of the far-field patterns of the scattered acoustic waves. This problem is difficult to solve since it is both nonlinear and improperly posed. We solve this problem by using two new methods. The first approach is analogous to the method developed by Kirsch and Kress, (cf. [14, 15, 16, 19]; see also [2]). The second approach is patterned after the method discovered by Colton and Monk (cf. $[6,7,8,9,16]$ ). The main idea of these methods is to stabilize the inverse problem by formulating it as a nonlinear optimization problem. Most of the previous solutions of the impedance inverse problem require a direct scattering problem to be solved at each step of the iteration scheme, (cf. $[4,5,17,18]$ ). However, it is possible to avoid this disadvantage by using either the Colton-Monk method or the Kirsch-Kress method.

Let us describe more precisely the problem under consideration. Let $D \subset R^{3}$ be a bounded connected domain with $C^{2}$ boundary $\partial D$, and let $n$ denote the unit outward normal to $\partial D$. Assume that an incoming time harmonic acoustic wave $u^{i}(x)=e^{i k(\hat{x}, x)}$ is scattered by the domain $D$ having an unknown continuous surface impedance $\lambda(x)$, $0 \leqq \lambda(x)$, for $x$ on the surface. Here, $(\hat{\alpha}, x)$ denotes the scalar or dot product between $\hat{\alpha}$ and $x$. The direct scattering problem (assuming $\lambda$ is known) is to find a solution of Helmholtz equation $u$ such that $u \in C^{2}\left(\mathbf{R}^{3} \backslash \bar{D}\right) \cap C^{1}\left(\mathbf{R}^{3} \backslash D\right)$ and

$$
\Delta_{3} u+k^{2} u=0 . \quad \text { in } \mathbf{R}^{3} \backslash D
$$

*This work was supported in part by the National Science Foundation. 


$$
\begin{gathered}
u(x)=e^{i k(x, \hat{\alpha})}+u^{s}(x) \\
\frac{\partial u}{\partial n}+i \lambda(x) u=0 \quad \text { on } \partial D \\
\lim _{r \rightarrow \infty} r\left(\frac{\partial u^{s}}{\partial r}-i k u^{s}\right)=0
\end{gathered}
$$

where $u^{s}$ denotes the scattered field, $r=|x|$, and the Sommerfeld radiation condition (1.4) is assumed to hold uniformly for $\hat{x}=x /|x|$ on the unit sphere $\partial \Omega$. The boundary condition (1.3) is called the impedance boundary condition. Physically, the surface impedance provides information on the physical properties of an obstacle. In particular, if $\lambda=0$ in (1.3) then the obstacle is acoustically hard, while if $\lambda=\infty$ in (1.3) then the obstacle is acoustically soft. It can be shown (cf. [5]), that under above assumptions there exists a unique solution of the boundary value problem (1.1)-(1.4), and the scattered wave $u^{\mathrm{s}}$ has the following asymptotic behaviour

$$
u^{s}(x)=\frac{e^{i k r}}{r} F(\hat{x} ; k, \hat{\alpha})+O\left(\frac{1}{r^{2}}\right)
$$

where the function $F$ is the far-field pattern corresponding to the incident wave $u^{i}$ with wave number $k$ and the direction of propagation $\hat{\alpha}$.

In this paper, we will discuss the following inverse scattering problem.

Definition 1.1. Inverse Problem: From knowledge of the domain $D$, and far-field patterns $F_{j}=F\left(\hat{x} ; k, \hat{\alpha}_{j}\right)$ corresponding to the incident plane waves $u_{j}^{i}$ with direction $\hat{\alpha}_{j}$, determine the function $\lambda$.

In [4], Colton and Kirsch proved that the surface impedance $\lambda$ is uniquely determined by a knowledge of the far-field pattern $F$ on some surface element of the unit sphere and fixed $k>0$. This uniqueness result is valid for all functions $\lambda$ such that the corresponding direct problem has a unique solution.

The contents of this paper is as follows. First, we solve the impedance inverse problem by using the method of Kirsch-Kress ([16]). Next, we present the ColtonMonk method. The method of Colton-Monk for the impedance inverse problem was originally presented in [7]. Our approach, (based on the ideas of [16]), is different from that of [7] and seems to be easier.

\section{The Kirsch-Kress method}

Let us now describe the Kirsch-Kress method for solving the inverse problem. Knowing the boundary of domain $D$ we can choose an auxiliary smooth closed surface $\Gamma$ contained in the domain $D$. For a technical reason we chose $\Gamma$ such that $k^{2}$ is not a 
Dirichlet eigenvalue of the Laplacian in the interior of $\Gamma$. This assumption involves no loss of generality since the choice of $\Gamma$ is arbitrary. Then we will try to find $u_{j}^{s}$ corresponding to the incident field $u_{j}^{i}, j=1, \ldots, n$, as an acoustic single-layer potential

$$
u_{j}^{s}(x)=\int_{\Gamma} \Phi(x, y) \phi_{j}(y) d s(y)
$$

with some unknown densities $\phi_{j} \in L^{2}(\Gamma), j=1, \ldots, n$, where

$$
\Phi(x, y)=\frac{\exp [i k|x-y|]}{4 \pi|x-y|}
$$

denotes the fundamental solution of the Helmholtz equation. It is easily seen that the single-layer potential satisfies the Sommerfeld radiation condition, (cf. [5]). It can also be shown, $([3,5])$ that

$$
u_{j}^{s}(x)=\frac{e^{i k|x|}}{4 \pi|x|} \int_{\Gamma} \phi_{j}(y) e^{-i k(\hat{x}, y)} d s(y)+O\left(\frac{1}{|x|^{2}}\right), \quad|x| \rightarrow \infty .
$$

Therefore, the far field patterns of the single layer potential are given by

$$
F_{j}(\hat{x} ; k, \hat{\alpha})=\frac{1}{4 \pi} \int_{\Gamma} \phi_{j}(y) e^{-i k(\hat{x}, y)} d s(y), \quad j=1, \ldots, n
$$

for $\hat{x}$ on the unit sphere $\partial \Omega$. Let us define the far-field pattern operator $\mathscr{F}: L^{2}(\Gamma) \rightarrow$ $L^{2}(\partial \Omega)$ by

$$
(\mathscr{F} \phi)(\hat{x})=\frac{1}{4 \pi} \int_{\Gamma} \phi(y) e^{-i k(\hat{x}, y)} d s(y), \quad \hat{x} \in \partial \Omega .
$$

Notice, that the operator $\mathscr{F}$ is a compact integral operator with an entire analytic kernel. Under the above assumption and notations, the inverse scattering problem leads us to the system of the first kind integral equations

$$
\mathscr{F} \phi_{j}=F_{j}
$$

for given (measured) far-field patterns $F_{j}, j=1,2, \ldots, n$.

We now consider the boundary condition (1.3). To this end, we introduce the boundary operator $B: L^{2}(\Gamma) \rightarrow L^{2}(\partial D)$ by

$$
(B \phi)(x)=\int_{\Gamma} \phi(y)\left[\frac{\partial}{\partial n_{x}} \Phi(x, y)+i \lambda(x) \Phi(x, y)\right] d s(y), \quad x \in \partial D
$$


Then the boundary condition (1.3) and the representation (2.1) imply

$$
B \phi_{j}+\left[\frac{\partial}{\partial n}+i \lambda\right] u_{j}^{i}=0
$$

where $u_{j}^{i}, j=1,2, \ldots, n$ are given incident plane waves corresponding to the scattered waves represented by the single layer potential. Obviously the boundary operator $B$ depends on the function $\lambda$. The main idea of our method for solving the inverse scattering problem, (which is analogous to the Kirsch-Kress method for the inverse Dirichlet problem for determining $\partial D$ ), is to assume that the scattered fields have representation (2.1) and then to solve the system of equations (2.5), (2.7) for the unknown functions $\lambda$ and $\phi_{j}, j=1, \ldots, n$.

Before further discussion of the system of equations (2.5), (2.7) we will investigate the basic properties of the operators $\mathscr{F}$ and $B$. Especially important from the point of view of solvability of the system (2.5), (2.7) is whether the operators $\mathscr{F}$ and $B$ are injective and have dense ranges. In fact, motivated by the results of [16], we have the following result:

Theorem 2.1. The far-field integral operator $\mathscr{F}: L^{2}(\Gamma) \rightarrow L^{2}(\partial \Omega)$ defined by (2.4) is injective and has dense range.

Proof. Let $\phi \in L^{2}(\Gamma)$ and $\phi=0$. Define the single layer potential $u^{s}$ with density $\phi$ given by the formula (2.1). Then the single layer potential satisfies the Sommerfeld radiation solution and its far-field pattern $\mathscr{F} \phi$ vanishes. Hence, $([5$, p. 78$])$, the singlelayer potential $u^{s}=0$ in the exterior of $\Gamma$. Denote by $\Gamma^{+}$the exterior of $\Gamma$ and by $\Gamma^{-}$the interior. The subscripts \pm denote the limits as $x$ tends to $\Gamma$ from outside and inside $\Gamma$ respectively. Now, the jump relation for the single layer-potential with $L^{2}$ densities, (cf. $[13,16])$, implies that $u^{-}=0$. Since the single layer potential $u^{s}$ satisfies the homogeneous Dirichlet problem in $\Gamma^{-}$and $k^{2}$ is not a Dirichlet eigenvalue of the Laplacian in $\Gamma^{-}$, we obtain $u^{s}=0$ in $\Gamma^{-}$. From the jump relation for the normal derivative of the single layer potential with $L^{2}$ densities (cf. $[13,16]$ )

$$
\frac{\partial u^{+}}{\partial n}-\frac{\partial u^{-}}{\partial n}=-\phi, \quad \text { on } \Gamma
$$

we conclude $\phi=0$ on the surface $\Gamma$, which is equivalent to injectivity of the far-field operator $\mathscr{F}$.

To prove that the operator $\mathscr{F}$ has a dense range we will consider its adjoint operator $\mathscr{F} *: L^{2}(\partial \Omega) \rightarrow L^{2}(\Gamma)$, given by

$$
\left(\mathscr{F}{ }^{*} g\right)(y)=\frac{1}{4 \pi} \int_{\partial \Omega} g(\hat{x}) e^{i k(\hat{x}, y)} d s(\hat{x}), \quad y \in \Gamma \subset \mathbf{R}^{3} .
$$

It is well known (cf. [16]), that for every bounded and linear operator $A: X \rightarrow Y$ we have 
$N\left(A^{*}\right)^{\perp}=\overline{R(A)}$ where $X, Y$ denote Hilbert spaces and, as usual, $N\left(A^{*}\right)$ is the null space for the operator $A^{*}$ and $R(A)$ denotes the range of $A$. Clearly, the operator $\mathscr{F}$ is bounded and linear and therefore if we prove that the null space of the operator $\mathscr{F}^{*}$ contains only the zero function then this would imply the second part of the theorem under consideration.

Let $\mathscr{F} * g=0$. Then the function

$$
v(y)=\frac{1}{4 \pi} \int_{\partial \Omega} g(\hat{x}) e^{i k(\hat{x}, y)} d s(\hat{x}), \quad y \in \mathbf{R}^{3}
$$

equals zero for $y \in \Gamma$ and hence solves the homogeneous Dirichlet problem in the interior of $\Gamma$ which implies that $v$ vanishes there. Since the function $v$ is analytic in $\mathbf{R}^{3}$ it follows that $v=0$ everywhere. But the function $v$ is a Herglotz wave function with kernel $g$ and vanishes everywhere. Therefore from [11] we deduce that $g=0$ in $L^{2}(\partial \Omega)$ which proves the injectivity of $\mathscr{F}^{*}$ and also completes the proof.

Now, by using the similar arguments to the above, we will establish the properties of the boundary operator $B$.

Theorem 2.2. The boundary operator $B: L^{2}(\Gamma) \rightarrow L^{2}(\partial D)$ defined by (2.6) is injective and has a dense range.

Proof. Let $\phi \in L^{2}(\Gamma)$ be such that

$$
B \phi=0 .
$$

Let us define the function

$$
u(x)=\int_{\Gamma} \phi(y) \Phi(x, y) d s(y), \quad x \in \mathbf{R}^{3} \backslash \Gamma
$$

Then the function $u$ is a solution of the Helmholtz euation in $\Gamma^{+}$satisfying the Sommerfeld radiation condition, (cf. [5]). Equation (2.11) implies the boundary condition

$$
\frac{\partial}{\partial n} u+i \lambda u=0, \quad \text { on } \partial D
$$

Recall that $0 \leqq \lambda$ on $\partial D$, and hence, by uniqueness of the solution for the exterior impedance boundary-value problem (cf. [5]), we conclude that $u=0$ in the exterior of $D$. Since the function $u$ is analytic in $\Gamma^{+}$, the unique continuation principle implies $u=0$ in $\Gamma^{+}$. From the jump relation for the single-layer potential with $L^{2}$ densities we obtain $u^{-}=0$. Since the single-layer potential $u$ also satisfies the Helmholtz equation in $\Gamma^{-}$and 
$k^{2}$ is not the Dirichlet eigenvalue for the Laplacian in $\Gamma^{-}$we have $u=0$ in $\Gamma^{-}$. The jump relation $(2.8)$ for the normal derivative of the single-layer potential with $L^{2}$ densities yields $\phi=0$, which proves the injectivity for the operator $B$.

Similar to the proof of Theorem 2.1 , to show that operator $B$ has a dense range consider the adjoint operator $B^{*}: L^{2}(\partial D) \rightarrow L^{2}(\Gamma)$ defined by

$$
\left(B^{*} \phi\right)(x)=\int_{\partial D} \phi(y)\left[\frac{\partial}{\partial n_{y}} \overline{\Phi(x, y)}-i \lambda(y) \overline{\Phi(x, y)}\right] d s(y), \quad x \in \Gamma
$$

We will show that the null space of $B^{*}$ is trivial. To establish this, let us consider the function $\phi$ such that

$$
B^{*} \phi=0
$$

and then using the funcion $\phi$, define

$$
u(x)=\int_{\partial D} \phi(y)\left[\frac{\partial}{\partial n_{y}} \overline{\Phi(x, y)}-i \lambda(y) \overline{\Phi(x, y)}\right] d s(y), \quad x \in \mathbf{R}^{3} \backslash D .
$$

The function $u$ is a solution of the Helmholtz equation in $D$. The condition (2.15) implies that $u=0$ on $\Gamma$, and hence, satisfies the homogeneous Dirichlet problem for the Helmholtz equation in $\Gamma^{-}$. Our choice of $\Gamma$ again implies that $u=0$ in $\Gamma^{-}$. Since the function $u$ is analytic in $D$, we conclude that $u=0$ in $D$. Taking the limit of $u(x)$ as $x$ tends to the boundary $\partial D$ from the interior and using the jump properties of the double and single layer potentials with square integrable densities (cf. [13]), we see that

$$
0=\phi(x)-2 \int_{\partial D} \phi(y)\left[\frac{\partial}{\partial n_{y}} \overline{\Phi(x, y)}-i \lambda(y) \overline{\Phi(x, y)}\right] d s(y)
$$

for $x \in \partial D$. From (2.17) we can deduce that $\phi \in C(\partial D)$. Denoting by the subscripts \pm the limits as $x$ tends to $\partial D$ from outside and inside $D$ respectively, using (2.16) and the fact that $\phi \in C(\partial D)$ we obtain the relations

$$
u^{+}-u^{-}=2 \phi, \quad \text { on } \partial D
$$

and

$$
\frac{\partial u^{+}}{\partial n}-\frac{\partial u^{-}}{\partial n}=2 i \lambda \phi, \quad \text { on } \partial D
$$

Since $u^{-}=\partial u^{-} / \partial n=0$ we have 


$$
\frac{\partial u^{+}}{\partial n}-i \lambda u^{+}=0, \quad \text { on } \partial D
$$

By the uniqueness of the solution to the exterior impedance problem, we have $u^{+}=0$ and hence, from (2.18) we obtain $\phi=0$. Therefore, the null space of the operator $B^{*}$ is trivial and this implies the theorem.

We now consider the problem of determining $\lambda, \phi_{1}, \ldots, \phi_{n}$ from the equations

$$
\mathscr{F} \phi_{j}=F_{j}
$$

and

$$
B \phi_{j}+\left[\frac{\partial}{\partial n}+i \lambda\right] u_{j}^{i}=0
$$

where, $F_{j}, u_{j}$ are given functions and the operators $\mathscr{F}$ and $B$ are defined by the formulas (2.4) and (2.6) respectively. Recall also that the operator $B$ depends on the function $\lambda$. Note also that since the integral operator $\mathscr{F}$ is compact and has an analytic kernel, the system of first kind integral equations (2.21) is severely ill-posed. Hence we need to regularize the problem. To this end, we assume that the function $\lambda$ belongs to a compact subset $V_{c}$ where

$$
V_{c}:=\left\{\lambda: \lambda \in C^{1, \beta}(\partial D),\|\lambda\|_{1, \beta} \leqq c, 0 \leqq \lambda \text { on } \partial D, 0 \leqq \beta \leqq 1\right\}
$$

where the constants $c, \beta$ represent a priori information and $C^{1, \beta}$ denotes the space of Hölder continuously differentiable functions on $\partial D$. Now define the functional $\mu:\left(L^{2}(\Gamma)\right)^{n} \times V_{c} \rightarrow \mathbf{R}$ by

$$
\begin{aligned}
\mu(\phi, \lambda ; F, \alpha):= & \sum_{j=1}^{n}\left\{\left\|\mathscr{F} \phi_{j}-F_{j}\right\|_{L^{2}(\partial \Omega)}^{2}+\alpha\left\|\phi_{j}\right\|_{L^{2}(\Gamma)}^{2}\right. \\
& \left.+\left\|B \phi_{j}+\left[\frac{\partial}{\partial n}+i \lambda\right] u_{j}^{i}\right\|_{L^{2}(\partial D)}^{2}\right\}
\end{aligned}
$$

where $\phi=\left(\phi_{1}, \ldots, \phi_{n}\right), F=\left(F_{1}, \ldots, F_{n}\right)$ and $\alpha>0$ is given regularization parameter. Now we can formulate the inverse scattering problem as the nonlinear optimization problem.

Definition 2.1. By an $\alpha$-optimal solution of the inverse scattering problem we understand the functions $\phi \in\left(L^{2}(\Gamma)\right)^{n}, \lambda \in V_{c}$, such that

$$
\mu(\phi, \lambda ; F, \alpha)=M(F, \alpha)=\inf \left\{\mu(\psi, h ; F, \alpha): \psi \in\left(L^{2}(\Gamma)\right)^{n}, h \in V_{c}\right\}
$$


Theorem 2.3. For each $\alpha>0$, the optimization problem (2.25), has a solution.

Proof. Let $\left(\phi^{(m)}, \lambda^{(m)}\right) \in\left(L^{2}(\Gamma)\right)^{n} \times V_{c}$ be a minimizing sequence, i.e.,

$$
\lim _{m \rightarrow \infty} \mu\left(\phi^{(m)}, \lambda^{(m)} ; F, \alpha\right)=M(F, \alpha)
$$

Since $V_{c}$ is a compact subset, we may choose $\lambda^{(m)}$ such that $\lambda^{(m)} \rightarrow \lambda \in V_{c}$ as $m \rightarrow \infty$. Hence,

$$
\alpha\left\|\phi_{j}^{(m)}\right\|_{L^{2}(\Gamma)}^{2} \leqq \mu\left(\phi^{(m)}, \lambda^{(m)}, F, \alpha\right) \rightarrow M(F, \alpha) \quad m \rightarrow \infty, j=1, \ldots, n,
$$

and, since $\alpha>0$, we see that the sequence $\left\{\phi_{j}^{(m)}\right\}$ is bounded, so we may assume weak convergence

$$
\phi_{j}^{(m)} \rightarrow \phi_{j} \in L^{2}(\Gamma)
$$

Since the operator $\mathscr{F}$ is compact, we have

$$
\mathscr{F} \phi_{j}^{(m)} \rightarrow \mathscr{F} \phi_{j}
$$

We will also show the above property for the operator $B$. Let us recall that the operator $B$ is compact and depends on the function $\lambda$, i.e., $B=B_{\lambda}$. Hence, for every $\varepsilon \geqq 0$ and $m$ sufficiently large

$$
\left\|B_{\lambda} \phi_{j}^{(m)}-B_{\lambda} \phi_{j}\right\| \leqq \frac{\varepsilon}{2}, \quad j=1, \ldots, n
$$

We can choose $m$ such that

$$
\left\|B_{\lambda^{(m)}} \phi_{j}^{(m)}-B_{\lambda} \phi_{j}^{(m)}\right\|=\left\|i \int_{\partial D} \phi_{j}^{(m)}(y) \Phi(x, y)\left[\lambda^{(m)}(x)-\lambda(x)\right] d s(y)\right\| \leqq \frac{\varepsilon}{2}
$$

for $x \in \partial D$ and $j=1, \ldots, n$. Using (2.30), (2.31) and the triangle inequality, we obtain

$$
B_{\lambda(m)} \phi_{j}^{(m)} \rightarrow B_{\lambda} \phi_{j}, \quad j=1, \ldots, n .
$$

From (2.24), (2.26), (2.29) and (2.32) we conclude that

$$
\lim _{m \rightarrow \infty} \sum_{j=1}^{n}\left\|\phi_{j}^{(m)}\right\|^{2} \leqq \sum_{j=1}^{n}\left\|\phi_{j}\right\|^{2}
$$

However, from (2.28) and (2.33) it follows that we have norm convergence 


$$
\lim _{m \rightarrow \infty} \phi_{j}^{(m)}=\phi_{j}, \quad j=1, \ldots, n
$$

Finally, using (2.26) and the continuity of the functional $M$, we have

$$
\lim _{m \rightarrow \infty} \mu\left(\phi^{(m)}, \lambda ; F, \alpha\right)=\mu(\phi, \lambda ; F, \alpha)=M(F, \alpha)
$$

which completes the proof.

Theorem 2.4. Assume that $F$ is the exact far-field pattern of the inverse scattering problem such that $\lambda$ is described by some function from $V_{c}$. Then

$$
\lim _{\alpha \rightarrow 0} \mu(\phi, \lambda ; F, \alpha)=0
$$

Proof. Since the operator $B$ has dense range, by Theorem 2.2 , for every given $\varepsilon>0$ there exists $\phi \in\left(L^{2}(\Gamma)\right)^{n}$ such that

$$
\sum_{j=1}^{n}\left\|B \phi_{j}+\left[\frac{\partial}{\partial n}+i \lambda\right] u_{j}^{i}\right\|_{L^{2}(\partial D)}^{2}<\varepsilon
$$

Due to the well-posedness of the direct scattering problem, the far-field pattern of radiating solutions of the Helmholtz equation depend continuously on the boundary data. Therefore we can estimate

$$
\left\|\mathscr{F} \phi_{j}-F_{j}\right\|^{2} \leqq K\left\|B \phi_{j}+\left[\frac{\partial}{\partial n}+i \lambda\right] u_{j}^{i}\right\|, \quad j=1, \ldots, n
$$

for some constant $K$. From (2.38) and the facts that $F$ and $\lambda$ satisfy the assumptions of Theorem 2.4 we have

$$
\mu(\phi, \lambda ; F, \alpha) \leqq(1+n K) \varepsilon+\alpha\|\phi\|_{\left(L^{2}(\Gamma)\right)^{n}}^{2} \rightarrow(1+n K) \varepsilon, \quad \text { as } \alpha \rightarrow 0 .
$$

Since $\varepsilon$ is arbitrary, (2.39) implies the theorem.

\section{The Colton-Monk method}

In this section we will investigate a different method for solving the inverse scattering problem under consideration. For the inverse Dirichlet problem of determining $\partial D$, this method was introduced by Colton and Monk, and is discussed in detail in $[6,7,10$; (see also 16)]. In this section, as in the previous, we consider the problem stated by the 
equations (1.1)-(1.4) and the Definition 1.1. We now make the assumption that $\lambda(x)>0$ for $x \in \partial D$.

We begin by noting that if $F(\hat{x} ; k, \hat{\alpha})$ is the far-field pattern of (1.1)-(1.4) corresponding to the incident wave $u^{i}$, then from Green's formula and the asymptotic behaviour of the fundamental solution of the Helmholtz equation (cf. [5]), we have

$$
F(\hat{x} ; k, \hat{\alpha})=\frac{1}{4 \pi} \int_{\partial D}\left\{u(y) \frac{\partial}{\partial n_{y}} e^{-i k(\hat{x}, y)}-\frac{\partial}{\partial n_{y}} u(y) e^{-i k(\hat{x}, y)}\right\} d s(y) .
$$

From (3.1) amd (1.4), by interchanging the order of integration we obtain

$$
\int_{\partial \Omega} F(\hat{x} ; k, \hat{\alpha}) g(\hat{x}) d s(\hat{x})=\frac{1}{4 \pi} \int_{\partial D} u(y ; k, \hat{\alpha})\left[\frac{\partial}{\partial n}+i \lambda(y)\right] v(y) d s(y)
$$

where

$$
v(y)=\int_{\partial \Omega} g(\hat{x}) e^{-i k(y, \hat{x})} d s(\hat{x})
$$

It is easily shown (see [7]) that if the unique solution of

$$
\begin{gathered}
\Delta_{3} v+k^{2} v=0 \quad \text { in } D \\
\left(\frac{\partial}{\partial n}+i \lambda\right) v=\left(\frac{\partial}{\partial n}+i \lambda\right) \Phi(\cdot, 0) \quad \text { on } \partial D
\end{gathered}
$$

has the form of a Herglotz wave function (3.3), then we have

$$
\int_{\partial \Omega} F(\hat{x} ; k, \hat{\alpha}) g(\hat{x}) d s(\hat{x})=1, \quad \hat{x} \in \partial \Omega
$$

for all angles $\hat{\alpha}$. The relation (3.6) now motivates us to introduce the far-field pattern operator $\mathrm{F}: L^{2}(\partial \Omega) \rightarrow L^{2}(\partial \Omega)$ by

$$
(\mathrm{Fg})(\hat{\alpha})=\int_{\partial \Omega} F(\hat{x} ; k, \hat{\alpha}) g(\hat{x}) d s(\hat{x}), \quad \hat{x} \in \partial \Omega
$$

Then, we can rewrite (3.6) as the integral equation

$$
\mathrm{Fg}=1
$$

The boundary condition (3.5) leads us to define the boundary value operator $H: L^{2}(\partial \Omega) \rightarrow L^{2}(\partial D)$ by 


$$
(H g)(x)=\int_{\partial \Omega}\left[\frac{\partial}{\partial n_{x}}+i \lambda(x)\right] e^{-i k(x, \hat{y})} g(\hat{y}) d s(\hat{y}), \quad x \in \partial D
$$

Then the condition (3.5) becomes

$$
H g=\left[\frac{\partial}{\partial n}+i \lambda\right] \Phi(\cdot, 0)
$$

The equations (3.8) and (3.10) are our basic tools for the solution of the inverse scattering problem. We first examine the properties of the operators $\mathrm{F}$ and $H$. Motivated by the ideas of [16], we have the following theorem:

Theorem 3.1. The far-field operator F, defined by (3.7), is injective and has dense range.

Proof. Let $\mathrm{Fg}=0$. Then we have

$$
\int_{\partial \Omega} F(\hat{x} ; k, \hat{\alpha}) g(\hat{x}) d s(\hat{x})=0, \quad x \in \partial \Omega .
$$

The reciprocity relation, (cf. [1]),

$$
F(\hat{x} ; k, \hat{\alpha})=F(-\hat{\alpha} ; k,-\hat{x})
$$

implies that

$$
\int_{\partial \Omega} F(\hat{x} ; k, \hat{\alpha}) g(-\hat{\alpha}) d s(\hat{\alpha})=0
$$

for all $\hat{x} \in \partial \Omega$. By the superposition principle, we note that the left-hand side of (3.13) is the far-field pattern of the scattered field $w^{5}$ corresponding to the Helmholtz equation with boundary condition $w^{s}=-\tilde{v}$ on $\partial D$, where $\tilde{v}$ is given by (3.3.) with $g(\hat{x})$ replaces by $g(-\hat{x})$. By (3.3), $w^{s}=0$ in $\mathbf{R}^{3} \backslash D$ and consequently $\tilde{v}=0$ on $\partial D$. The result of [11] implies that $g(-\hat{x})=0$ and hence $g(\hat{x})=0$ for $\hat{x} \in \partial \Omega$ which proves that the operator $F$ is injective. Almost the same argument shows that adjoint operator $\mathrm{F}^{*}$ is injective and this implies the density of the range of the operator $F$.

Now, we consider the operator $H$.

Theorem 3.2. The operator $H$, defined by (3.9), is injective and has dense range.

Proof. Let $H g=0$. Then define the function 


$$
v(x)=\int_{\partial \Omega} g(\hat{y}) e^{-i k(x, \hat{y})} d s(\hat{y}), \quad x \in \mathbf{R}^{3} .
$$

The function $v$ is a solution of the Helmholtz equation in $\mathbf{R}^{3}$. We have

$$
\frac{\partial v}{\partial n}+i \lambda v=0 \quad \text { on } \partial D
$$

Since $\lambda(x)>0$ for $x \in \partial D$ we have $v=0$ in $D$ and, by unique continuation, for $x \in \mathbf{R}^{3}$. By the results of [11] we can conclude that $g=0$ in $L^{2}(\partial \Omega)$. Now, let us consider the adjoint operator of $H, H^{*}: L^{2}(\partial D) \rightarrow L^{2}(\partial \Omega)$

$$
\left(H^{*} \psi\right)(\hat{y})=\int_{\partial D}\left[\frac{\partial}{\partial n_{x}}-i \lambda(x)\right] e^{-i k(x, \hat{y})} \psi(x) d s(x) .
$$

Assume that $H^{*} \phi=0$ in $L^{2}(\partial \Omega)$ and consider the function

$$
u^{s}(y)=\int_{\partial D}\left[\frac{\partial}{\partial n_{x}}-i \lambda(x)\right] \overline{\Phi(x, y)} \phi(x) d s(x)
$$

The far-field pattern of the function $u^{s}$ is given by $1 /(4 \pi) H^{*} \phi=0$. Hence, $u^{s}=0$ in $\mathbf{R}^{3} \backslash D$ and also

$$
\int_{\partial D}\left[\frac{\partial}{\partial n_{x}}-i \lambda(x)\right] \overline{\Phi(x, y)} \phi(x) d s(x)=0 \quad \text { on } \Gamma .
$$

Now, using exactly the same arguments as in the proof of Theorem 2.2 (except interchange the roles of interior and exterior domains), we conclude that $\phi=0$ and hence the operator $H^{*}$ is injective which implies the theorem.

Based on the equations (3.8) and (3.10) we are now able to formulate our optimization problem for solving the inverse scattering problem. We first define the functional

$$
\eta(g, \lambda ; F, \rho)=\|\mathrm{Fg}-1\|_{L^{2}(\partial \Omega)}^{2}+\left\|H g-\left[\frac{\partial}{\partial n}+i \lambda\right] \Phi(\cdot, 0)\right\|_{L^{2}(\partial D)}^{2}
$$

simultaneously, over all $\lambda \in V_{c}$ and $g \in U_{\rho}=\left\{g \in L^{2}(\partial \Omega):\|g\|_{L^{2}(\partial \Omega)} \leqq \rho\right\}$.

Definition 3.1. The pair $(\lambda, g), \lambda \in V_{c}, g \in U_{\rho}$ is a $\rho$-optimal solution of the inverse scattering problem if 


$$
\eta(g, \lambda ; F, \rho)=\inf \left\{\eta(q, r ; F, \rho): q \in U_{\rho}, r \in V_{c}\right\}
$$

where $\rho>0$ is a regularization parameter.

Theorem 3.3. For each $\rho>0$ the optimization problem (3.20) has a solution.

Proof. Let $\left(g_{n}, \lambda_{n}\right)$ be a minimizing sequence for (3.20). Since the set $V_{c}$ is compact, we can choose the sequence $\lambda_{n}$ such that

$$
\lambda_{n} \rightarrow \lambda
$$

strongly and

$$
g_{n} \rightarrow g
$$

converges in a weak sense. The functions $\lambda \in V_{c}$ since the set $V_{c}$ is compact. Also, we have

$$
\|g\|^{2}=\lim _{n \rightarrow \infty}\left(g, g_{n}\right) \leqq\|g\| \rho
$$

and hence, $g \in U_{\rho}$. Since the operators $H$ and $\mathrm{F}$ are compact, it follows that $\mathrm{Fg} g_{n} \rightarrow \mathrm{Fg}$ and $H_{\lambda_{n}} g_{n} \rightarrow H_{\lambda} g$ which concludes the proof.

Theorem 3.4. Let $F$ be the far-field pattern such that the function $\lambda \in V_{\mathrm{c}}$. Then

$$
\lim _{\rho \rightarrow \infty} \eta(g, \lambda ; F, \rho)=0 .
$$

Proof. Let $\varepsilon>0$. Since the operator $H$ has a dense range, we can find a function $g_{\varepsilon}$ such that

$$
\left\|H g_{\varepsilon}-\left[\frac{\partial}{\partial n}+i \lambda\right] \Phi(\cdot, 0)\right\|_{L^{2}(\partial D)}^{2} \leqq \varepsilon .
$$

Now we will show that

$$
\left\|\mathrm{Fg} g_{\varepsilon}-1\right\|_{L^{2}(\Omega)}^{2} \leqq C \varepsilon
$$

where $C$ is a constant depending on the function $u$, the solution of (1.1)-(1.4). It follows from (3.7), that

$$
\mathrm{F} g_{\varepsilon}-1=\int_{\partial \Omega} F(\hat{x} ; k, \hat{\alpha}) g_{\varepsilon}(\hat{x}) d s(\hat{x})-1
$$


As in the proof of (3.6), elementary calculation shows that

$$
\int_{\partial \Omega} F(\hat{x} ; k, \hat{\alpha}) g_{\varepsilon}(\hat{x}) d s(\hat{x})-1=\int_{\partial D} \frac{\partial u}{\partial n}\left(H g_{\varepsilon}-\left[\frac{\partial}{\partial n_{x}}+i \lambda\right] \Phi(x, 0)\right) d s(y) .
$$

The Schwarz inequality gives

$$
\left|\mathrm{F} g_{\varepsilon}-1\right|^{2} \leqq \int_{\partial D}\left|\frac{\partial}{\partial n} u(y)\right|^{2} d s(y) \varepsilon^{2} \leqq C_{1} \varepsilon^{2}
$$

where $\int_{\partial D}|(\partial / \partial n) u(y)|^{2} d s(y) \leqq C_{1}$, which implies (3.26). From (3.25) and (3.26) we have

$$
\eta\left(g_{\varepsilon}, \lambda ; F, \rho\right) \leqq(1+C) \varepsilon
$$

Since the constant $\varepsilon$ is an arbitrary positive number, (3.30) implies the theorem.

Acknowledgement. This work is a portion of the author's dissertation [12] written at the University of Delaware under the direction of Professor David L. Colton, to whom the author is very greatly indebted.

\section{REFERENCES}

1. T. S. Angell, D. Colton and R. Kress, Far field patterns and inverse scattering problems for imperfectly conducting obstacles, Math. Proc. Cambridge Philos. Soc. 106 (1989), 553-569.

2. T. Angell, R. Klejman and G. Roach, An inverse transmission problem for the Helmholtz equation, Inverse Problems 3 (1987), 149-180.

3. D. Colton, Partial Differential Equations (Random House, New York, 1989).

4. D. Colton and A. KIRSCH, The determination of the surface impedance of an obstacle from measurements of the far field pattern, SIAM J. Appl. Math. 41, (1981), 8-15.

5. D. Colton and R. KREss, Integral Equation Methods in Scattering Theory (John Wiley, New York, 1983).

6. D. Colton and P. Monk, A novel method for solving the inverse scattering problem for time harmonic acoustic waves in the resonance region, SIAM J. Appl. Math. 45 (1985), 1039-1053.

7. D. Colton and P. Monk, A novel method for solving the inverse scattering problem for time harmonic acoustic waves in the resonance region II, SIAM J. Appl. Math. 46 (1986), 506-523.

8. D. Colton and P. Monk, The inverse scattering problem for time acoustic waves in an inhomogeneous medium, Quart. J. Mech. Appl. Math. 41 (1988), 97-125.

9. D. Colton and P. Monk, The inverse scattering problem for time harmonic acoustic waves in an inhomogeneous medium: numerical experiments, IMA J. Appl. Math. 42 (1989), 77-95.

10. D. Colton and P. Monk, The numerical solution of the three dimensional inverse scattering problem for time harmonic acoustic waves. SIAM J. Sci. Statist. Comput. 8 (1987), 278-291.

11. P. Hartman and C. Wilcox, On solutions of the Helmholtz equation in exterior domains, Math. Z. 75 (1961), 228-255. 
12. A. KęDzierawski, Inverse scattering problems for acoustic waves in an inhomogeneous medium (Ph.D. dissertation, University of Delaware, Newark, DE, 1990).

13. H. Kersten, Grenz- und Sprungrelationen für Potentiale mit quadrat-summier-barer Flächenbelegung, Resultate Math. 3 (1980), 17-24.

14. A. KIRSCH and R. Kress, An optimalization method in inverse acoustic scattering, in: Boundary Elements IX, Vol. 3. Fluid Flow and Potential Applications (C. A. Brebbia, W. L. Wendland and G. Kuhn, eds, Springer-Verlag, Heidelberg, 1987) 3-18.

15. A. KIRSCH, R. Kress, P. Monk and A. ZINN, Two methods for solving the inverse acoustic scattering problem, Inverse Problems 4 (1988), 749-770.

16. R. KRESs, Linear Integral Equations (Springer-Verlag, New York, 1989).

17. R. T. SMITH, An inverse scattering problem for an obstacle with an impedance boundary condition, J. Math. Anal. Appl. 105, (1985), 333-356.

18. R. T. SMiтh, A stable method for an inverse problem in acoustic scattering by an obstacle with an impedance boundary condition, Proc. Roy. Soc. Edinburgh 98A (1984), 335-364.

19. A. ZiNN, On an optimization method for the full- and the limited-aperature problem in inverse acoustic scattering for a sound-soft obstacle, Inverse Problems 5 (1989), 239-253.

Department of Mathematics

Delaware State College

DOVER, DE 19901

U.S.A. 